\title{
USING TWO OPTIONS FOR CONJUGATING WAVE FIELDS IN THE METHOD OF PARTIAL DOMAINS
}

\author{
V. T. Grinchenko ${ }^{1}$, V. O. Husak ${ }^{2}$, V. T. Matsypura ${ }^{2}$
}

${ }^{1}$ Institute of Hydromechanic of NAS Ukraine, The National Academy of Sciences of Ukraine, Kyiv, Ukraine, E-mail: grinchenko@nas.gov.ua

${ }^{2}$ Faculty of Mechanics and Mathematics, Taras Shevchenko National University of Kyiv, Kyiv, Ukraine, E-mail: gusakw7@gmail.com, mnivtt@gmail.com

\section{ВИКОРИСТАННЯ ДВОХ ВАРІАНТІВ СПРЯЖЕННЯ ХВИЛЬОВИХ ПОЛІВ В МЕТОДІ ЧАСТКОВИХ ОБЛАСТЕЙ}

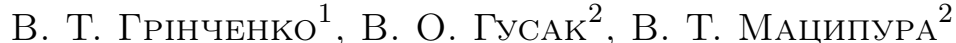

${ }^{1}$ Інститут гідромеханіки НАН України, Національна академія наук України, Київ, Україна, E-mail: grinchenko@nas.gov.ua

${ }^{2}$ Механіко-математичний факультет, Київський національний університет імені Тараса Шевченка, Київ, Україна, E-mail: gusakw7@gmail.com, mnivtt@gmail.com

ABstract. The paper considers the use of two options of conjugation of wave fields in the method of partial domains: the first is the conjugation of wave fields in the sense of the root mean square approximation, the second is the pointwise conjugation of wave fields. A solution is obtained for the problem of radiation from one or two flat pistons into a wedge-shaped waveguide. It is shown that with the same number of modes in partial domains, the version of the root mean square approximation is more accurate. A quite acceptable calculation accuracy was obtained for the option of pointwise conjugation of fields at a certain value of the wave distance between the nodal points.

KEYWORDS: partial domains method, wedge-shaped waveguide, wave field.

АнотАцІя. В роботі розглянуто використання двох варіантів спряження хвильових полів в методі часткових областей: перший спряження хвильових полів в сенсі середньоквадратичного наближення, другий - поточкове спряження хвильових полів. Отримано розв'язок задачі про випромінювання одного або двох плоских поршнів у клинуватий хвилевод. Показано, що при однаковій кількості мод в часткових областях, варіант середньоквадратичного наближення є більш точним. Отримано цілком прийнятну точність розрахунку для варіанту поточкового спряження полів при певній величині хвильової відстані між вузловими точками.

КлЮчовІ СЛовА: метод часткових областей, клинуватий хвилевод, хвильове поле. 


\section{ВСтуП}

Згідно з методом часткових областей [1] вся складна (неканонічна) область існування хвильового поля розбивається на прості області, в кожній з яких вдається побудувати загальний розв'язок рівняння Гельмгольца.

Після цього виникає необхідність спряження цих розв'язків на границях часткових областей i, при необхідності, задоволення граничних умов на певних граничних поверхнях та поверхнях випромінювачів. Реалізація граничних умов призводить до рівнянь з невідомими коефіцієнтами, які $\epsilon$ амплітудними коефіцієнтами мод у відповідних часткових областях. В ці рівняння входять функції від просторових змінних, оскільки деяка границя описується просторовою змінною (або декількома змінними), яка змінюється у визначених межах.

Для визначення шуканих амплітудних коефіцієнтів мод слід перейти від цих рівнянь до нескінченної системи лінійних алгебраїчних рівнянь, яка далі розв'язується методом редукції. Варіант переходу до лінійної алгебраїчної системи рівнянь, який можна назвати класичним, спирається на побудовані загальні розв'язки для рівняння Гельмгольца в часткових областях [1].

Загальний розв'язок передбачає наявність в ньому повної та ортогональної системи функцій, завдяки якій можна записати у вигляді ряду Фур'є будь-яку (розумну з фізичної точки зору) функцію. Використовуючи ортогональність цього набору функцій можна отримати систему алгебраїчних рівнянь. Про цю процедуру говорять як про наближення функцій в розумінні мінімальності середньоквардатичного відхилення.

Такий підхід добре досліджений і випробуваний. Де може виникнути складність в його реалізації? Вона може виникнути в ситуації, якщо для запису хвильових полів в часткових областях доводиться використовувати кілька (причому, часто, різних) систем координат. Як результат, при переході до алгебраїчної системи рівнянь можуть виникнути складні інтеграли. Часто, їх аналітичне обчислення неможливо, та і чисельне обчислення може бути пов'язане з певними труднощами.

Інший варіант переходу до системи алгебраїчних рівнянь полягає в прирівнюванні функцій (полів в суміжних часткових областях) в кінцевому наборі точок (назвемо їх вузловими точками) на границі цих областей. Кількість вузлових точок на границі визначає порядок системи рівнянь i, тим самим, кількість шуканих амплітудних коефіцієнтів мод в часткових областях. Як бачимо, такий підхід не містить обчислювальних проблем. Однак виникають два питання.

Перше, наскільки варіант поточкового спряљсення полів на границі часткових областей поступається класичному середнъоквадратичному наближенню при реалізачї̈ граничних умов?

Друге, яка хвильова відстань між точками спряження полів забезпечить прийнятну точність обчислень?

Мета даної статті - дати відповіді на поставлені питання. 


\section{1. ВИПРОМІнювАНнЯ ПОРШНЯ в Клин}

Щоб отримати орієнтири у відповідях на поставлені питання, звернемося до задачі випромінювання поршня в нескінченний клинуватий хвилевод (рис. 1). Задача плоска, тобто відсутня залежність хвильового поля від однієї з просторових координат (на рис. 1 це координата $z$ декартової системи координат $x O y$ ). Поверхні хвилеводу вважаємо ідеально жорсткими, тобто, дорівнює нулю нормальна складова коливальної швидкості на цих поверхнях.

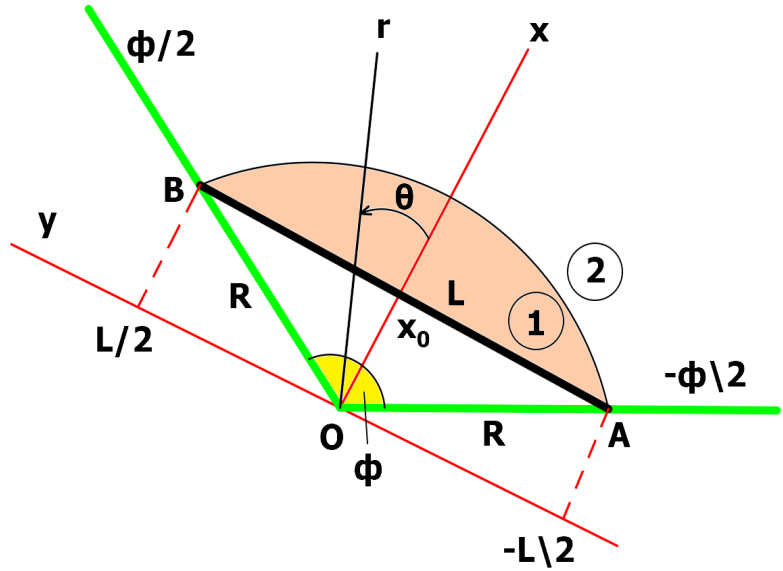

Рис 1. Геометрія задачі випромінювання плоского поршня у клинуватий хвилевод

Згідно рис. $1, R$ - радіус дуги, $L$ - довжина поршня $A B, \phi-$ розхил клину. Для опису полів в часткових областях слід використовувати дві системи координат: декартову (координати $x O y$ ) і полярну (координати $r O \theta)$. Зв'язок між декартовими і полярними координатами визначається формулами: $x=r \cos \theta, y=r \sin \theta ; r=\sqrt{x^{2}+y^{2}}, \tan \theta=y / x$.

Поршень здійснює гармонічні коливання з частотою $\omega$. На поверхні поршня задається амплітуда коливальної швидкості $v\left(x=x_{0}, y\right)$. Нехай розподіл швидкості на поверхні поршня є симетричним відносно середини поршня. Областю існування поля $є$ внутрішність клина при координаті $x \geq x_{0}$. Будемо вважати, що в трикутній області $O A B$ хвильове поле відсутнє. Згідно методу часткових областей виділимо дві часткові області, границі яких утворюють частини координатних поверхонь використовуваних систем координат.

Перша часткова область обмежена поверхнею поршня і дугою кола радіуса $R,-\phi / 2 \leq \theta \leq \phi / 2$, тобто, утворена частинами поверхонь декартової і полярної систем координат відповідно. Друга часткова область задається полярними координатами $r \geq R,-\phi / 2 \leq \theta \leq \phi / 2$.

Комплексну амплітуду тиску в області 1 запишемо у вигляді суми полів плоскопаралельного і клинуватого хвилеводів (часовий множник $\exp (-i \omega t)$ 
не пишемо):

$$
p_{1}=\sum_{n=1}^{\infty} A_{n} \cos \left(\alpha_{n} y\right) \exp \left(i \gamma_{n}\left(x-x_{0}\right)\right)+\sum_{n=1}^{\infty} B_{n} \cos \left(\beta_{n} \theta\right) \frac{J_{\beta_{n}}(k r)}{J_{\beta_{n}}^{\prime}(k R)},
$$

де $\alpha_{n}=\frac{2(n-1) \pi}{L}, \gamma_{n}=\sqrt{k^{2}-\alpha_{n}^{2}}, \quad \beta_{n}=\frac{2(n-1) \pi}{\phi}, n=1,2 \ldots ; \quad J_{\beta_{n}}(k r)-$ функція Бесселя, штрих при функції Бесселя означає похідну по повному аргументу, $k=2 \pi / \lambda-$ хвильове число, $\lambda$ - довжина хвилі.

Поле тиску в області 2 представимо у вигляді суперпозиції біжучих циліндричних хвиль:

$$
p_{2}=\sum_{n=1}^{\infty} C_{n} \cos \left(\beta_{n} \theta\right) \frac{H_{\beta_{n}}^{(1)}(k r)}{H_{\beta_{n}}^{(1)^{\prime}}(k R)}
$$

де $H_{\beta_{n}}^{(1)}(k r)-$ функція Ханкеля.

Для визначення трьох наборів невідомих коефіцієнтів $A_{n}, B_{n}, C_{n}, n=$ $1,2 \ldots$ слід розписати три граничні умови:

$$
\begin{aligned}
\text { 1) } \frac{1}{i \omega \rho} \frac{\partial p_{1}}{\partial x} & =v\left(x_{0}, y\right), \quad x=x_{0}, \quad y=\left[-\frac{L}{2}, \frac{L}{2}\right], \\
\text { 2) } p_{1} & =p_{2}, \quad r=R, \quad \theta=\left[-\frac{\phi}{2}, \frac{\phi}{2}\right], \\
\text { 3) } \frac{\partial p_{1}}{\partial r} & =\frac{\partial p_{2}}{\partial r}, \quad r=R, \quad \theta=\left[-\frac{\phi}{2}, \frac{\phi}{2}\right] .
\end{aligned}
$$

Підставивши вирази (1) і (2) в умови (3)-(5) приходимо до трьох рівнянь, в які входять нескінченні ряди. Функції, які входять в ці рівняння, залежать від просторових координат, їх границі зміни вказані в умовах (3)-(5).

$$
\begin{aligned}
& \text { 1) } i \sum_{n=1}^{\infty} A_{n} \gamma_{n} \cos \left(\alpha_{n} y\right)+\sum_{n=1}^{\infty} B_{n} \frac{\partial}{\partial x}\left[\cos \left(\beta_{n} \arctan \frac{y}{x}\right) \times\right. \\
& \left.\times \frac{J_{\beta_{n}}\left(k \sqrt{\left.x^{2}+y^{2}\right)}\right.}{J_{\beta_{n}}^{\prime}(k R)}\right]_{x=x_{0}}=i v\left(x_{0}, y\right) \\
& \text { 2) } \sum_{n=1}^{\infty} A_{n}\left[\cos \left(\alpha_{n} R \sin \theta\right) \exp \left(i \gamma_{n}\left(R \cos \theta-x_{0}\right)\right)\right]+ \\
& +\sum_{n=1}^{\infty} B_{n} \cos \left(\beta_{n} \theta\right) \frac{J_{\beta_{n}}(k R)}{J_{\beta_{n}}^{\prime}(k R)}-\sum_{n=1}^{\infty} C_{n} \cos \left(\beta_{n} \theta\right) \frac{H_{\beta_{n}}{ }^{(1)}(k R)}{H_{\beta_{n}}^{(1)^{\prime}}(k R)}=0, \\
& \text { 3) } \sum_{n=1}^{\infty} A_{n} \frac{\partial}{\partial r}\left[\cos \left(\alpha_{n} r \sin \theta\right) \exp \left(i \gamma_{n}\left(r \cos \theta-x_{0}\right)\right)\right]_{r=R^{+}}+ \\
& +\sum_{n=1}^{\infty} B_{n} \cos \left(\beta_{n} \theta\right)-\sum_{n=1}^{\infty} C_{n} \cos \left(\beta_{n} \theta\right)=0 .
\end{aligned}
$$


Перехід від рівнянь (6)-(8) до нескінченної системи лінійних алгебраїчних рівнянь можна провести двома шляхами:

- перший - застосовуючи середьноквадратичне наближення при виконанні граничних умов,

- другий - поточкове спряження полів на границі.

Розв'язок нескінченної системи лінійних алгебраїчних рівнянь будемо проводити методом редукції, контролюючи при цьому в ході обчислень якість виконання граничних умов. Для цього обмежимо нескінченні ряди у виразах (6)-(8), задаючи кількість коефіцієнтів $A_{n}, B_{n}, C_{n}$ відповідно $N_{1}, N_{2}, N_{3}$. Оскільки амплітудні коефіцієнти $B_{n}$ і $C_{n}$ пов'язані з власними функціями одного і того ж клинуватого хвилеводу, то логічно покласти їх кількості однаковими, тобто $N_{2}=N_{3}$.

\section{Середнвоквадратичне наближення}

При такому підході, помножимо скалярно вираз (6) на функції $\cos \left(\alpha_{m} y\right)$, $m=1,2, \ldots, N_{1}$, а вирази (7) і (8) - на функції $\cos \left(\beta_{m} \theta\right), m=1,2, \ldots, N_{2}$. При цьому використаємо властивість ортогональності функцій $\cos \left(\alpha_{m} y\right)$, $m=1,2, \ldots, N_{1}$ на відрізку $y=[-L / 2, L / 2]$, і властивість ортогональності функцій $\cos \left(\beta_{m} \theta\right), m=1,2, \ldots, N_{2}$ на відрізку $\theta=[-\phi / 2, \phi / 2]$. В результаті приходимо до системи лінійних алгебраїчних рівнянь:

$$
\begin{aligned}
& \sum_{n=1}^{N_{1}} A_{n} i \gamma_{n} L \delta_{m n}+\sum_{n=1}^{N_{2}} B_{n} G_{m n}^{(1)}+\sum_{n=1}^{N_{2}} C_{n} \cdot 0=i V_{m}, \quad m=1,2, \ldots, N_{1}, \\
& \sum_{n=1}^{N_{1}} A_{n} G_{m n}^{(2)}+\sum_{n=1}^{N_{2}} B_{n} \frac{J_{\beta_{n}}(k R)}{J_{\beta_{n}}^{\prime}(k R)} \phi \delta_{m n}-\sum_{n=1}^{N_{2}} C_{n} \frac{H_{\beta_{n}}^{(1)}(k R)}{H_{\beta_{n}}^{(1)^{\prime}}(k R)} \phi \delta_{m n}=0, \\
& m=1,2, \ldots, N_{2}, \\
& \sum_{n=1}^{N_{1}} A_{n} G_{m n}^{(3)}+\sum_{n=1}^{N_{2}} B_{n} \phi \delta_{m n}-\sum_{n=1}^{N_{2}} C_{n} \phi \delta_{m n}=0, \quad m=1,2, \ldots, N_{2}, \\
& \text { де } G_{m n}^{(1)}=\int_{-L / 2}^{L / 2} \frac{\partial}{\partial x}\left[\cos \left(\beta_{n} \arctan \frac{y}{x}\right) \frac{J_{\beta_{n}}\left(k \sqrt{x^{2}+y^{2}}\right)}{J_{\beta_{n}}^{\prime}(k R)}\right]_{x=x_{0}} \cos \left(\alpha_{m} y\right) d y, \\
& G_{m n}^{(2)}=\int_{-\phi / 2}^{\phi / 2} \cos \left(\alpha_{n} R \sin \theta\right) \exp \left(i \gamma_{n}\left(R \cos \theta-x_{0}\right)\right) \cos \left(\beta_{m} \theta\right) d \theta, \\
& G_{m n}^{(3)}=\int_{-\phi / 2}^{\phi / 2} \frac{\partial}{\partial r}\left[\cos \left(\alpha_{n} r \sin \theta\right) \exp \left(i \gamma_{n}\left(r \cos \theta-x_{0}\right)\right)\right]_{r=R} \cos \left(\beta_{m} \theta\right) d \theta,
\end{aligned}
$$




$$
\delta_{m n}= \begin{cases}1, & m=n=1 \\ 0.5, & m=n>1, \quad V_{m}=\int_{-L / 2}^{L / 2} v\left(x_{0}, y\right) \cos \left(\alpha_{m} y\right) d y \\ 0, & m \neq n\end{cases}
$$

\section{Поточкове спряљення полів на граничі}

Позначимо координати точок спряження (вузлових точок) на поверхні поршня $\left(x_{m}=x_{0}, y_{m}\right)$, а на границі розділу часткових областей 1 і $2-$ $\left(r_{m}=R, \theta_{m}\right)$.

Вибравши координати вузлових точок $\left(x_{m}=x_{0}, y_{m}\right)$ i $\left(r_{m}=R, \theta_{m}\right)$, запишемо вирази (3)-(5) у вигляді системи лінійних алгебраїчних рівнянь:

$$
\begin{gathered}
i \sum_{n=1}^{N_{1}} A_{n} \gamma_{n} \cos \left(\alpha_{n} y_{m}\right)+\sum_{n=1}^{N_{2}} B_{n} \frac{\partial}{\partial x}\left[\cos \left(\beta_{n} \arctan \frac{y_{m}}{x}\right) \times\right. \\
\left.\times \frac{J_{\beta_{n}}\left(k \sqrt{x^{2}+y_{m}^{2}}\right)}{J_{\beta_{n}}^{\prime}(k R)}\right]_{x=x_{0}}=i v\left(x_{0}, y_{m}\right), \quad m=1,2, \ldots, N_{1} ; \\
\sum_{n=1}^{N_{1}} A_{n}\left[\cos \left(\alpha_{n} R \sin \theta_{m}\right) \exp \left(i \gamma_{n}\left(R \cos \theta_{m}-x_{0}\right)\right)\right]+\sum_{n=1}^{N_{2}} B_{n} \cos \left(\beta_{n} \theta_{m}\right) \times \\
\times \frac{J_{\beta_{n}}(k R)}{J_{\beta_{n}}^{\prime}(k R)}-\sum_{n=1}^{N_{2}} C_{n} \cos \left(\beta_{n} \theta_{m}\right) \frac{H_{\beta_{n}}^{(1)}(k R)}{H_{\beta_{n}}^{(1)^{\prime}}(k R)}=0, \quad m=1,2, \ldots, N_{2} ; \\
\sum_{n=1}^{N_{1}} A_{n} \frac{\partial}{\partial r}\left[\cos \left(\alpha_{n} r \sin \theta_{m}\right) \exp \left(i \gamma_{n}\left(r \cos \theta_{m}-x_{0}\right)\right)\right]_{r=R}+ \\
+\sum_{n=1}^{N_{2}} B_{n} \cos \left(\beta_{n} \theta_{m}\right)-\sum_{n=1}^{N_{2}} C_{n} \cos \left(\beta_{n} \theta_{m}\right)=0, \quad m=1,2, \ldots, N_{2} .
\end{gathered}
$$

Відправною точкою в розрахунках є відношення відстані між вузловими точками $\triangle h$ вздовж поверхні поршня і на криволінійній границі часткових областей 1 і 2 до довжини хвилі $\lambda$, тобто величина $\triangle h / \lambda$. Виходячи з величини $\triangle h / \lambda$, визначається кількість вузлових точок вздовж поверхні поршня $N_{1}$ і на криволінійній границі $N_{2}$ :

$$
N_{1}=\left[\frac{L}{\triangle h}\right], \quad N_{2}=\left[\frac{\phi \cdot R}{\triangle h}\right] .
$$

Величини $N_{1}$ і $N_{2}$ визначають, відповідно, кількість мод плоскопаралельного хвилеводу (з характерним розміром $L$ ) і клинуватого хвилеводу (з розхилом $\phi$ ) для двух варіантів розв'язку задачі. Зрозуміло, що величини 
$N_{1}$ і $N_{2}$ задають порядок системи лінійних алгебраїчних рівнянь, розв'язок якої дозволяє знайти шукані амплітудні коефіцієнти мод $A_{n}, B_{n}, C_{n}$.

В якості контрольних величин використовуємо такі інтегральні критерії:

$$
\delta P^{s k}=\frac{P_{i}^{s k}-P_{d}^{s k}}{P_{i}^{s k}}, \quad \delta P^{t}=\frac{P_{i}^{t}-P_{d}^{t}}{P_{i}^{t}}, \quad \delta P=\frac{P_{i}^{s k}-P_{i}^{t}}{P_{i}^{s k}},
$$

де $P_{i}^{s k}$ і $P_{i}^{t}-$ середній потік потужності (або, коротше, потужність) з поверхні поршня при використанні середньоквадратичного наближення і при поточковому спряженні хвильових полів; $P_{d}^{s k}$ i $P_{d}^{t}-$ потужність в дальній зоні області 2 при використанні середньоквадратичного наближення і при поточковому спряженні хвильових полів.

Формула для середнього потоку потужності має вигляд [2]:

$$
P=0.5 \int_{(s)} \operatorname{Re}\left(p v_{n}^{*}\right) d S,
$$

де $p$ i $v_{n}^{*}$ - тиск і нормальна швидкість на деякій поверхні $S$, зірочка знак комплексного спряження.

Виберемо параметри задачі:

$$
R / \lambda=1.2, \quad \phi=150^{\circ},
$$

тоді

$$
L / \lambda=2.3 .
$$

Розподіл швидкості по поверхні поршня рівномірний, тобто $v\left(x_{0}, y\right)=1$. Результати розрахунків представлені в таблиці.

\begin{tabular}{|l|l|l|l|l|l|}
\hline$\triangle h / \lambda$ & $N_{1}$ & $N_{2}$ & Середньоквадр. $\delta P^{s k}$ & Точки $\delta P^{t}$ & $\delta P$ \\
\hline 0.6 & 4 & 6 & $2.3 \cdot 10^{-3}$ & -0.2 & -0.09 \\
0.5 & 5 & 7 & $1.25 \cdot 10^{-3}$ & -0.075 & -0.06 \\
0.45 & 6 & 7 & $1.2 \cdot 10^{-3}$ & -0.07 & -0.056 \\
0.4 & 6 & 8 & $7.4 \cdot 10^{-4}$ & -0.047 & -0.041 \\
0.35 & 7 & 9 & $5 \cdot 10^{-4}$ & -0.034 & -0.031 \\
0.3 & 8 & 11 & $3 \cdot 10^{-4}$ & -0.021 & -0.02 \\
0.25 & 10 & 13 & $1.6 \cdot 10^{-4}$ & -0.014 & -0.014 \\
0.2 & 12 & 16 & $9 \cdot 10^{-5}$ & -0.009 & -0.009 \\
0.15 & 16 & 21 & $4.5 \cdot 10^{-5}$ & -0.005 & -0.005 \\
0.1 & 24 & 32 & $3 \cdot 10^{-5}$ & -0.002 & -0.002 \\
\hline
\end{tabular}

Доповненням до таблиці служать графіки на рис. 2, на якому показані (зліва направо) графіки модулей тиску $|p|$ і швидкості $\left|v_{r}\right|$ вздовж границі розділу часткових областей 1,2 і модуль тиску $\left|p_{i}\right|$ на поверхні поршня. Верхній ряд відовідає хвильовій відстані між вузловими точками $\triangle h / \lambda=$ 0.5 , а нижній ряд $-\triangle h / \lambda=0.2 ;$ лінії - середньоквадратичне наближення, точки - поточкове спряження полів. 

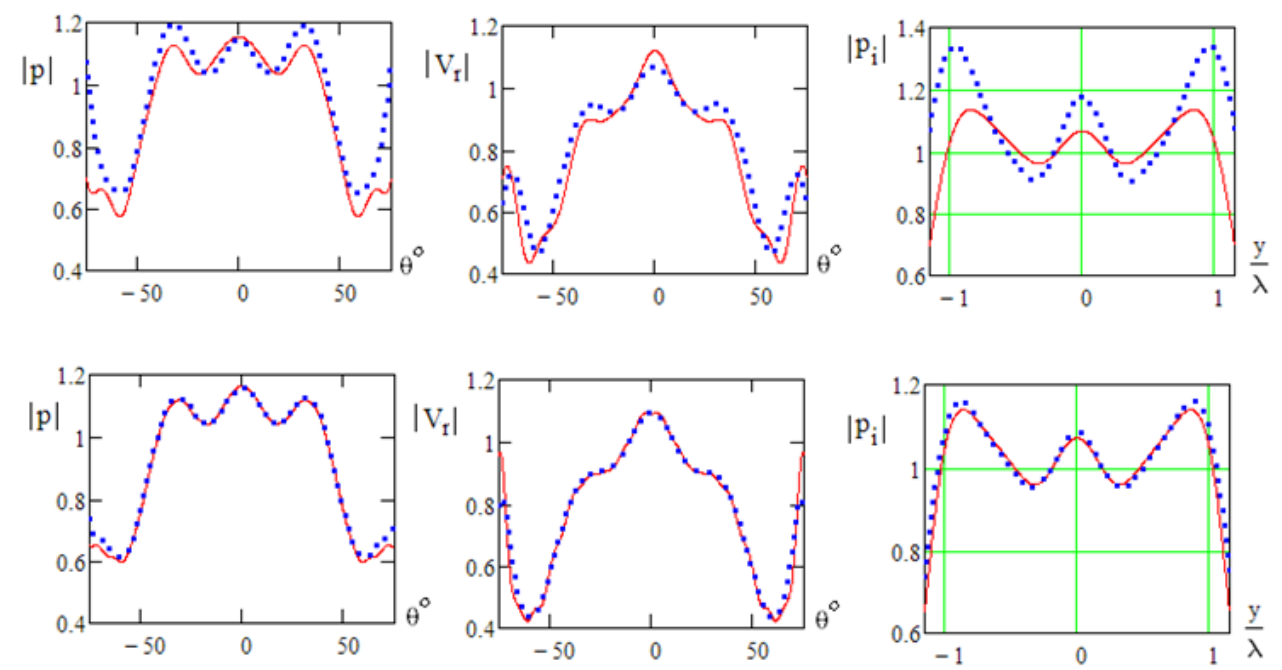

Рис 2. Графіки модулей тиску $|p|$ і швидкості $\left|v_{r}\right|$ вздовж границі розділу часткових областей 1,2 і модуль тиску $\left|p_{i}\right|$ на поверхні поршня. Верхній ряд відповідає хвильовій відстані між вузловими точками $\triangle h / \lambda=0.5$, а нижній ряд $-\triangle h / \lambda=0.2 ;$ лінї - середньоквадратичне наближення, точки - поточкове спряження полів

Дані таблиці і графіки на рис. 2 показують, що, як і слід було очікувати, середньоквадратичне наближення показує кращі результати при всіх значеннях величини $\triangle h / \lambda$. Однак при величині $\triangle h / \lambda<0.2$ отримуємо цілком прийнятну точність розрахунку і для методу поточкового спряження полів. Звісно, іншим фактором, який впливає на вибір величини $\triangle h / \lambda, \epsilon$ хвильові розміри розглянутої моделі.

\section{2. ВиПРОМІнювАНня ДВоХ ПОРшнІв в кЛИн}

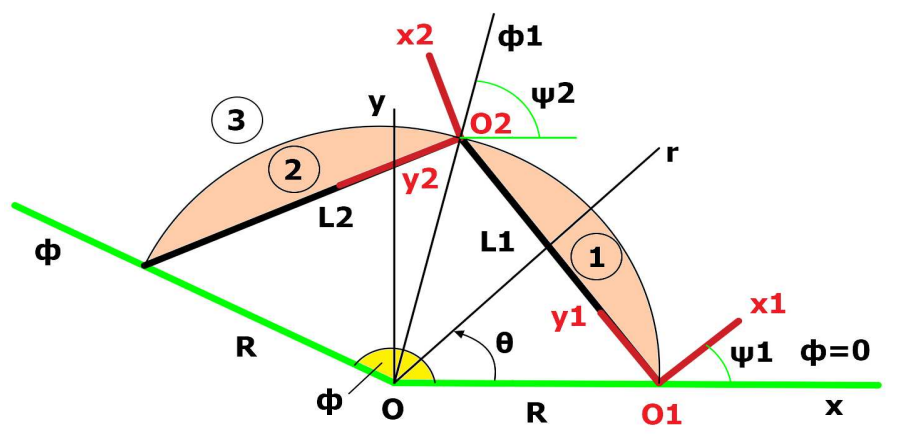

Рис 3. Геометрія задачі випромінювання двох плоских поршнів у клинуватий хвилевод

На рис. 3 показана геометрія задачі випромінювання двох плоских поршнів у клинуватий хвилевод. Будемо вважати, що довжина поршнів може 
бути різною, тому симетрія задачі відносно деякої осі відсутня. Крім декартової $x O y$ і полярної $r O \theta$ систем координат, введемо декартові системи координат $x_{1} O_{1} y_{1}$ i $x_{2} O_{2} y_{2}$. Положення всіх чотирьох систем координат показані на рис. 3.

Згідно рис. 3 вся область існування хвильового поля ділиться на три часткові області. Запишемо поля тиску в цих областях:

$$
p_{1}=\sum_{n=1}^{\infty} A_{n} \cos \left(\alpha_{n}^{(1)} y_{1}\right) \exp \left(i \gamma_{n}^{(1)} x_{1}\right)+\sum_{n=1}^{\infty} B_{n} \cos \left(\beta_{n}^{(1)} \theta\right) \frac{J_{\beta_{n}^{(1)}}(k r)}{J_{\beta_{n}^{(1)}}^{\prime}(k R)}
$$

де $\alpha_{n}^{(1)}=\frac{(n-1) \pi}{L_{1}}, \quad \gamma_{n}^{(1)}=\sqrt{k^{2}-\left(\alpha_{n}^{(1)}\right)^{2}}, \quad \beta_{n}^{(1)}=\frac{(n-1) \pi}{\phi_{1}}, \quad n=1,2, \ldots$;

$$
\begin{aligned}
& p_{2}=\sum_{n=1}^{\infty} C_{n} \cos \left(\alpha_{n}^{(2)} y_{2}\right) \exp \left(i \gamma_{n}^{(2)} x_{2}\right)+ \\
& +\sum_{n=1}^{\infty} D_{n} \cos \left(\beta_{n}^{(2)}\left(\theta-\phi_{1}\right)\right) \frac{J_{\beta_{n}^{(2)}}(k r)}{J_{\beta_{n}^{(2)}}^{\prime}(k R)}
\end{aligned}
$$

де $\alpha_{n}^{(2)}=\frac{(n-1) \pi}{L_{2}}, \quad \gamma_{n}^{(2)}=\sqrt{k^{2}-\left(\alpha_{n}^{(2)}\right)^{2}}, \quad \beta_{n}^{(2)}=\frac{(n-1) \pi}{\phi-\phi_{1}}, \quad n=1,2, \ldots$;

$$
p_{3}=\sum_{n=1}^{\infty} E_{n} \cos \left(\beta_{n}^{(3)} \theta\right) \frac{H_{\beta_{n}^{(3)}}^{(1)}(k r)}{H_{\beta_{n}^{(3)}}^{(1)^{\prime}}(k R)}
$$

де $\beta_{n}^{(3)}=\frac{(n-1) \pi}{\phi}, \quad n=1,2, \ldots$

Для визначення п'яти наборів невідомих коефіцієнтів $A_{n}, B_{n}, C_{n}, D_{n}$, $E_{n}, n=1,2, \ldots$ слід розписати п'ять граничних умов:

$$
\begin{aligned}
\text { 1) } \frac{1}{i \omega \rho} \frac{\partial p_{1}}{\partial x_{1}}=v_{1}\left(y_{1}\right), \quad x_{1}=0, \quad y_{1}=\left[0, L_{1}\right], \\
\text { 2) } \frac{1}{i \omega \rho} \frac{\partial p_{2}}{\partial x_{2}}=v_{2}\left(y_{2}\right), \quad x_{2}=0, \quad y_{2}=\left[0, L_{2}\right], \\
\text { 3) } p_{1}=p_{3}, \quad r=R, \quad \theta=\left[0, \phi_{1}\right], \\
\text { 4) } p_{2}=p_{3}, \quad r=R, \quad \theta=\left[\phi_{1}, \phi\right], \\
\text { 5) } \frac{\partial p_{3}}{\partial r}=\left\{\begin{array}{lll}
\frac{\partial p_{1}}{\partial r}, & r=R, & \theta=\left[0, \phi_{1}\right], \\
\frac{\partial p_{2}}{\partial r}, & r=R, & \theta=\left[\phi_{1}, \phi\right] .
\end{array}\right.
\end{aligned}
$$

Виходячи з величини $\triangle h / \lambda$, визначається кількість вузлових точок вздовж поверхні поршнів $N_{1}, N_{2}$ і на дузі кола радіусом $R-N_{3}, N_{4}$ : 


$$
N_{1}=\left[\frac{L_{1}}{\triangle h}\right], \quad N_{2}=\left[\frac{L_{2}}{\triangle h}\right], \quad N_{3}=\left[\frac{\phi_{1} \cdot R}{\triangle h}\right], \quad N_{4}=\left[\frac{\left(\phi-\phi_{1}\right) \cdot R}{\triangle h}\right]
$$

Величини $N_{1}$ і $N_{2}$ визначають, відповідно, кількість мод плоскопаралельного хвилеводу з характерним розміром $L_{1}$ i $L_{2}$, а величини $N_{3}$ i $N_{4}$ визначають кількість мод клинуватого хвилеводу з розхилом $\phi_{1}$ i $\left(\phi-\phi_{1}\right)$. Кількість мод в області 3 задамо у вигляді суми величин $N_{3}+N_{4}$.

Щоб розписати граничні умови (20)-(24) слід використати формули, які пов'язують кординати точки спостереження в різних системах координат (рис. 3):

$$
\begin{gathered}
x=r \cos \theta, \quad x=r \sin \theta, \quad r=\sqrt{x^{2}+y^{2}}, \quad \tan \theta=y / x, \\
x=x_{j} \cos \psi_{j}-y_{j} \sin \psi_{j}+a_{j}, \quad x_{j}=\left(x-a_{j}\right) \cos \psi_{j}+\left(y-b_{j}\right) \sin \psi_{j}, \\
y=x_{j} \sin \psi_{j}+y_{j} \cos \psi_{j}+b_{j}, \quad y_{j}=-\left(x-a_{j}\right) \sin \psi_{j}+\left(y-b_{j}\right) \cos \psi_{j} .
\end{gathered}
$$

В цих формулах індекс $j=1,2$, що відповідає декартовим системам координат $x_{1} O_{1} y_{1}$ i $x_{2} O_{2} y_{2} ; \psi_{j}$ - кут повороту системи координат $x_{j} O_{j} y_{j}$ відносно системи $x O y ; a_{j}, b_{j}$ - координати початку $O_{j}$ системи координат $x_{j} O_{j} y_{j}$ в системі декартових координат $x O y$.

Згідно рис. 3 координати точки $O_{1}-a_{1}=R, b_{1}=0$; координати точки

$$
O_{2}-a_{2}=R \cos \phi_{1}, \quad b_{2}=R \sin \phi_{1} .
$$

Кути повороту систем координат $x_{j} O_{j} y_{j}, j=1,2$ відносно системи $x O y$ визначаються по формулам:

$$
\psi_{1}=\frac{\phi_{1}}{2}, \quad \psi_{2}=\frac{\phi_{1}+\phi}{2} .
$$

Наявність формул (26), (27) дозволяе провести необхідне перетворення при підстановці виразів (17)-(19) в умови (20)-(24). Внаслідок об'ємності отримані формули не виписуємо.

Задамо наступні параметри моделі:

$$
R / \lambda=0.8, \quad \phi=150^{\circ}, \quad \phi_{1}=90^{\circ},
$$

тоді

$$
L_{1} / \lambda=1.13, \quad L_{2} / \lambda=0.8, \quad \psi_{1}=45^{\circ}, \quad \psi_{2}=120^{\circ} .
$$

Розподіл швидкості по поверхні обох поршнів рівномірний, тобто $v_{1}\left(y_{1}\right)=$ 1 i $v_{2}\left(y_{2}\right)=1$. Виберемо хвильову відстань між вузловими точками $\triangle h / \lambda=$ 0.1. При даних параметрах моделі отримуємо, що

$$
N_{1}=12, \quad N_{2}=9, \quad N_{3}=13, \quad N_{4}=9, \quad N_{5}=N_{3}+N_{4}=22 .
$$



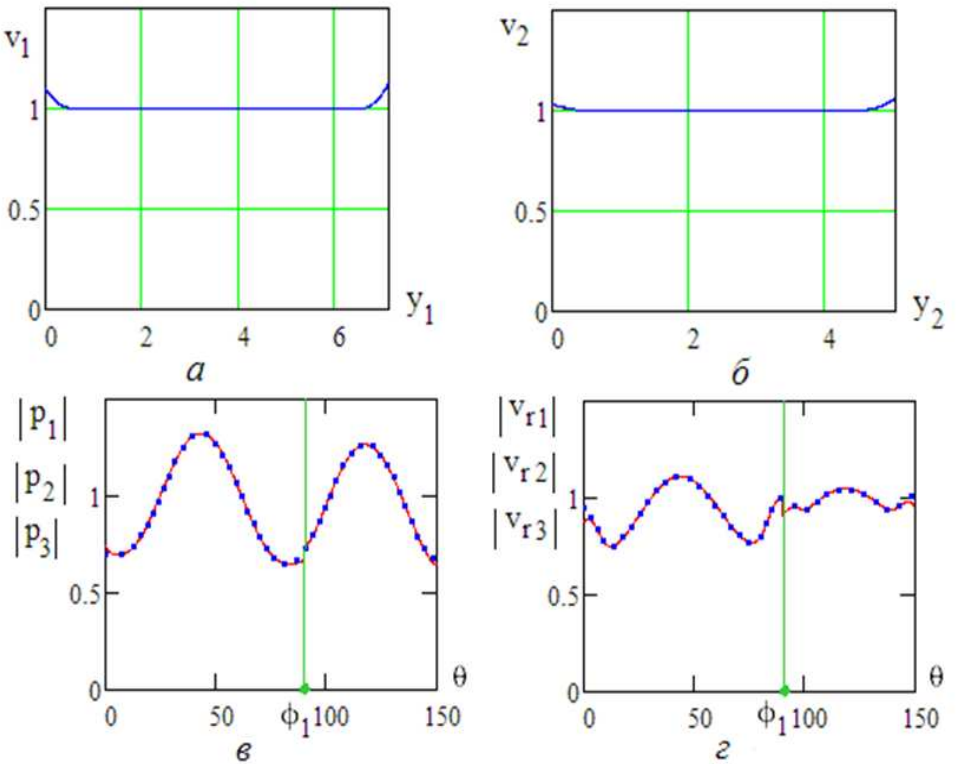

Рис 4. Графічна ілюстрація виконання граничних умов: $a, \sigma$ - амплітуда швидкості на поверхні першого і другого поршнів; модулі тиску (в) i модулі радіальної швидксоті (2) вздовж дуги кола радіусом $R$ : лінія - зі сторони областей 1 і 2 , точки - зі сторони області 3

На рис. 4 представлена графічна ілюстрація виконання граничних умов: $a, \sigma$ - амплітуда швидкості на поверхні першого і другого поршнів; модулі тиску (в) і модулі радіальної швидкості (2) вздовж дуги кола радіусом $R$ : лінія - зі сторони областей 1 і 2, точки - зі сторони області 3.

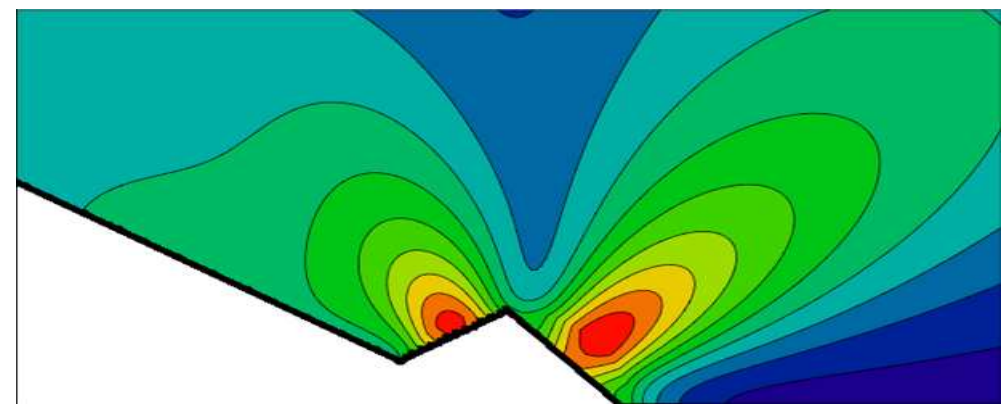

Рис 5. Поле модуля тиску в околі двох випромінювачів у клинуватому хвилеводі, $R / \lambda=0.8, \phi=150^{\circ}, \quad \phi_{1}=90^{\circ}, L_{1} / \lambda=1.13, L_{2} / \lambda=0.8$

На рис. 5 показано поле модуля тиску в околі двох випромінювачів у клинуватому хвилеводі. Якщо покласти $\phi=180^{\circ}$, то виникає можливість визначити поле випромінювання чотирьох поршнів розташованих у вигляді прямокутника у вільному просторі. 


\title{
Висновки
}

В статті на базі методу часткових областей отримано розв'язок задачі про випромінювання одного або двох плоских поршнів в нескінченний клин. Даний підхід дозволяє, подібним чином, побудувати розв'язок і для випадку кількості поршнів більше двох.

При спряженні хвильових полів на границях часткових областей використано два варіанти: перший - спряження полів в сенсі середньоквадратичного наближення, другий - поточкове спряження хвильових полів.

Показано, що при одинаковій кількості мод в часткових областях для двох варіантів спряження полів, варіант середньоквадратичного наближення є більш точним.

Показано, що при величині хвильової відстані між вузловими точками $\triangle h / \lambda<0.2$ отримаємо цілком прийнятну точність розрахунку і для методу поточкового спряження полів. Звісно, іншим фактором, який впливає на вибір величини $\triangle h / \lambda$, є хвильові розміри розглянутої моделі.

\section{ЛiтеРАТУРА}

1. Grinchenko V. T., Vovk I. V., Matsypura V. T. Acoustic wave problems. New York: Begell House, Inc., 2018. 439 p.

2. Грінченко В. Т., Вовк I. В., Маципура В. Т. Основи акустики. Київ: Наукова думка, 2007. $640 \mathrm{c}$.

Надійшла: 23.06.2021 / Прийнята: 10.09.2021

\section{ИСПОЛЬЗОВАНИЕ ДВУХ ВАРИАНТОВ СОПРЯЖЕНИЯ ВОЛНОВЫХ ПОЛЕЙ В МЕТОДЕ ЧАСТИЧНЫХ ОБЛАСТЕЙ}

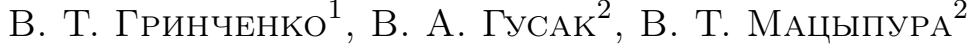 \\ ${ }^{1}$ Институт гидромеханики НАН Украины, Национальная академия наук Украины, \\ Киев, Украина, E-mail: grinchenko@nas.gov.ua \\ ${ }^{2}$ Механико-математический факультет, Киевский национальный университет имени \\ Тараса Шевченка, Киев, Украина, E-mail: gusakw7@gmail.com, mnivtt@gmail.com
}

АннотАция. В работе рассмотрено использование двух вариантов сопряжения волновых полей в методе частичных областей: первый - сопряжение волновых полей в смысле среднеквадратичного приближения, второй - поточечное сопряжения волновых полей. Получено решение задачи об излучении одного или двух плоских поршней в клиновидный волновод. Показано, что при одинаковом количестве мод в частичных областях, вариант среднеквадратичного приближения является более точным. Получено вполне приемлемую точность расчета для варианта поточечного сопряжения полей при определенной величине волнового расстояния между узловыми точками.

КЛЮчЕВЫЕ СЛОВА: метод частичных областей, клиновидный волновод, волновое поле. 